\title{
Percepción de esfuerzo durante el ejercicio: ¿Es válida su medición en la población infantil?
}

\author{
IVÁN RODRÍGUEZ N.*,**,***,***** y DARWIN GATICA S.***,****,*****
}

\section{Perceived exertion during exercise: is their measurement valid in children?}

The perceived exertion (PE) corresponds to the subjective sensation caused, in part, by the metabolic changes during exercise. This variable has been widely used during exercise tests and during training processes and physical rehabilitation in healthy and chronically diseased children. Additionally, it has been established that the assessment of PE properties (validity and reliability) is strongly determined by the type of scale, the applied paradigm and the cognitive development. Evidence is overwhelming in establishing the validity of this measurement in children aging from 4 years old, by using adapted scales. At 8 years-old the application of estimate-production paradigm is suggested and from 13 to 15 years-old the Borg scale 6-20 would be used. In Chile EPInfant scale has been developed for measuring child perceived exertion. This scale has shown an acceptable performance in incremental exercise test in healthy children aged 8 to 15 years old, justifying its use. We review the assessment of PE properties in children, considering key aspects in the design of scales and cognitive development of children. Additionally, the evidence regarding the validity of the Chilean scale EPInfant in children is presented. Finally the research currently under development and its projections on this issue are discussed.

Key words: Validity of tests, children, cognition, exercise, exercise testing, physical exertion, perceived exertion, dyspnea.

\section{Resumen}

La percepción de esfuerzo (PE) corresponde a la valoración subjetiva causada, en parte, por los cambios metabólicos durante el ejercicio. Esta variable ha sido ampliamente utilizada en pruebas de ejercicio y rehabilitación física en niños sanos y con enfermedades crónicas. Adicionalmente, ha sido establecido que las propiedades de medición (validez y confiabilidad) de la PE son fuertemente determinadas por el tipo de escala, paradigma de aplicación y desarrollo cognitivo. En este contexto, ha sido demostrado que la medición de la PE es válida en niños a partir de los 4 años a través de escalas adaptadas. Desde los 8 años es factible la utilización de esta variable mediante el paradigma de estimación-producción y desde los 13 a 15 años mediante escalas de adultos v.gr. Borg 6-20. En nuestro país, ha sido desarrollada la escala de medición de esfuerzo percibido infantil (EPInfant), la cual, ha mostrado, un rendimiento aceptable durante pruebas de ejercicio incremental en niños sanos de entre 8-15 años de edad, justificando su utilización en nuestro medio. En esta revisión se discuten las propiedades de medición de la PE en población infantil, considerando aspectos esenciales en el diseño de las escalas y el desarrollo cognitivo de los niños. Adicionalmente, se presenta la evidencia existente respecto a la validez de la escala EPInfant en niños de nuestro país. Finalmente se discuten las líneas de investigación actualmente en desarrollo que determinarán las perspectivas en esta temática.

Palabras clave: Validez de los test, niños, cognición, ejercicio, prueba de ejercicio, esfuerzo físico, percepción de esfuerzo, disnea.

\footnotetext{
Conflicto de interés: Los autores declaran no poseer conflicto de interés.

* Escuela de Kinesiología, Facultad de Ciencias de la Salud, Universidad San Sebastián Concepción, Chile.

** Departamento de Fisiologia Cardiovascular e Respiratória, Escola Paulista de Medicina, Universidade Federal de São Paulo, Brasil.

*** Programa de Magíster y Doctorado en Ciencias Médicas, Facultad de Medicina, Universidad de la Frontera, Temuco, Chile.

**** Escuela de Kinesiología, Facultad de Salud, Universidad Santo Tomás, Concepción, Chile.

*****Grupo de Investigación en Salud Cardiovascular y Respiratoria (IDEAS-CVR), Concepción, Chile.
} 


\section{Introducción}

La percepción de esfuerzo (PE) corresponde a la valoración subjetiva causada, en parte, por los cambios metabólicos durante el ejercicio. A menudo, esta variable ha sido asociada con la percepción subjetiva de dificultad respiratoria (disnea), no obstante, la PE constituye una configuración de sensaciones que vincula de manera integrada (además de la disnea) el estrés y fatiga del sistema muscular, cardiovascular y respiratorio durante el ejercicio ${ }^{1,2}$.

Los primeros estudios en este campo fueron realizados a finales de los ' 50 e inicios de los '60 por Gunnar Borg, quien desarrolló el primer instrumento de evaluación (homónimo) para cuantificar la $\mathrm{PE}^{3-5}$. La escala de Borg posee 15 categorías numéricas (6-20) y descriptores verbales que representan el nivel de intensidad del ejercicio ejecutado ${ }^{6}$ (Figura 1A). El rango de descriptores numéricos de esta escala fue establecido en interdependencia con la frecuencia cardíaca (FC) de un sujeto adulto durante el ejercicio con el fin de estimar el estrés fisiológico desde una simple operación aritmética a partir de la PE (FC $=\mathrm{PE} \times 10)^{7}$. Más tarde fue desarrollada la escala “category ratio 0-10” (CR-10) (Figura 1B), la cual, presenta propiedades de razón/proporción y permite un procesamiento estadístico más elaborado $^{7}$. Las propiedades de medición de estas escalas han sido ampliamente estudiadas en adultos, lo que ha permitido respaldar su utilización en distintos contextos ${ }^{8}$.

Pese a que el inicio de los estudios de la PE se remonta a mediados del siglo $\mathrm{XX}$, recién en los años '70 fueron realizadas las primeras observaciones de la PE en población infantil. Oded Bar-Or y cols., demostraron por primera vez en niños que la PE incrementa de manera lineal con el ascenso de la FC durante el ejercicio de intensidad incremental ${ }^{9}$. Posteriormente, los escasos estudios que se realizaron para determinar las propiedades psicométricas de las escalas de Borg en niños, mostraron resultados conflictivos ${ }^{10-14}$.

El primer intento por generar una escala especialmente adaptada para niños fue llevado a cabo por Nystad y cols (1989), quienes reemplazaron los 9 descriptores verbales de la escala de Borg (6-20) por ilustraciones representativas de fatiga asociada con intensidad de ejercicio (escala RPE-C). Sin embargo, esta adaptación mostró una escasa validez de criterio concurrente en estudios posteriores ${ }^{15,16}$. Un avance significativo en el estudio de la PE en niños fue realizado por Eston y cols. quienes, 5 años más tarde, desarrollaron la escala CERT (Children Effort Rating Table), constituyendo el primer instrumento para la medición del esfuerzo percibido diseñado exclusivamente para niños (Figura 1C). Esta escala presenta un rango de categorías numéricas más intuitivo (1-10) y expresiones verbales de esfuerzo ampliamente entendidas por los niños y adolescentes ${ }^{17,18}$. A partir de la escala CERT y su ulterior versión ilustrada, la escala PCERT (Pictorial Children Effort Rating Table) $)^{19-21}$; una serie de instrumentos fueron desarrollados, los cuales, han mostrado distintos niveles de validez y confiabilidad en población pediátrica de distintas edades y modalidades de ejercicio $^{22}$.

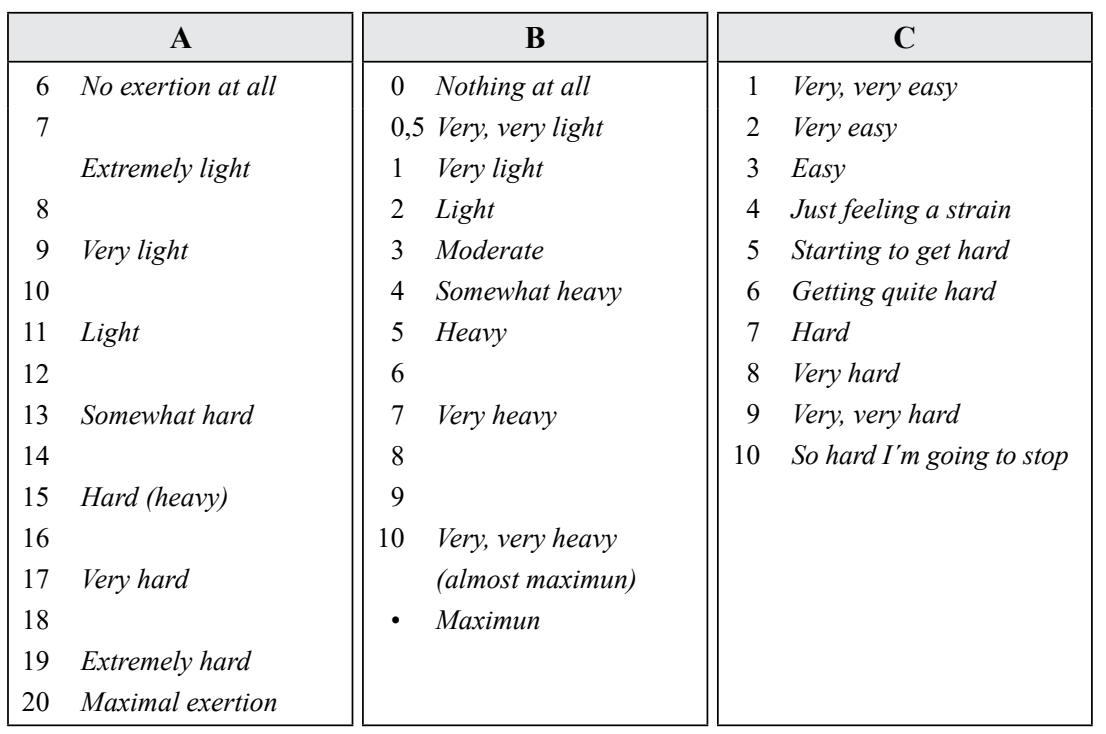

Figura 1. Escalas de medición de esfuerzo percibido. En A y B se muestran los instrumentos desarrollados por Borg (modificado de referencia 7) (A escala RPE 6-20; B escala CR-10). En $\mathrm{C}$ se muestra la escala CERT (Children effort rating table) desarrollada por Eston y cols (modificado de referencia 17). 
En la presente revisión se discutirá las propiedades de medición de la PE en población infantil, considerando aspectos esenciales en el diseño de las escalas construidas y el desarrollo cognitivo de los niños. Adicionalmente, se presenta la metodología de aplicación de esta variable en la población infantil de nuestro país. Finalmente serán discutidas las líneas de investigación actualmente en desarrollo que van a determinar las perspectivas en esta área de estudio.

\section{Propiedades psicométricas}

Hoy en día es ampliamente aceptado que las propiedades de medición (validez y confiabilidad) de la PE son fuertemente determinadas por el tipo de escala, paradigma de aplicación y desarrollo cognitivo.

\section{Tipo de escala}

Recientemente Rodríguez y cols., mediante una revisión sistemática y meta-análisis determinaron la validez asociada a criterio de las escalas de medición de la PE en niños sanos durante una prueba de ejercicio incremental estandarizada. Los resultados confirmaron lo establecido en revisiones (no sistemáticas) realizadas previamente $e^{1,22}$, al revelar que aquellas escalas que presentan ilustraciones gráficas para población infantil presentan una mayor validez de criterio concurrente que las escalas construidas para adultos, como la escala de Borg 6-20 y CR-1022. Incluso la escala CERT (Figura 1C) presentó un menor rendimiento de medición que las escalas ilustradas para niños. Vale la pena destacar que la escala RPE-C, que corresponde a la escala de Borg 6-20 con ilustraciones, mostró un bajo nivel de validez de criterio probablemente debido a la distribución de sus categorías numéricas (6-20), las cuales, serían poco intuitivas para la población infantil. Estos resultados confirman que, tanto la distribución numérica de las categorías, como la existencia de ilustraciones en la matriz gráfica de las escalas van a determinar las propiedades del instrumento de medición ${ }^{22}$. En la Tabla 1 se muestra el índice de validez de criterio de las escalas de PE más ampliamente estudiadas en niños.

\section{Paradigmas de aplicación y desarrollo cognitivo}

En términos generales es posible describir dos modalidades de aplicación de la PE, modalidad estimación y modalidad estimación-producción $^{2,23}$. El primer paradigma se refiere a la medi- ción de la PE durante el ejercicio, el cual, ha sido dosificado previamente a partir de algún parámetro fisiológico ( $\mathrm{FC}$, consumo de $\mathrm{O}_{2}: \mathrm{VO}_{2} \mathrm{u}$ otro) ${ }^{23}$. Éste corresponde al paradigma de aplicación de la PE más ampliamente utilizado en el contexto de la rehabilitación pulmonar infantil en nuestro país $^{24-27}$. Por su parte, la modalidad estimaciónproducción corresponde a la prescripción de una determinada intensidad de ejercicio en función de la $\mathrm{PE}$ hasta alcanzar un cierto nivel de $\mathrm{FC}, \mathrm{VO}_{2}$ o lactato sanguíneo (modalidad producción); el cual, ha sido reportado en un test de ejercicio incremental realizado previamente (modalidad estimación) ${ }^{28,29}$.

Estos paradigmas o modelos exigen la integración de tres dimensiones: perceptual/psicológico, fisiológico y situacional, por lo tanto, la posibilidad de utilizar uno u otro paradigma depende estrechamente del desarrollo cognitivo del individuo ${ }^{1}$. Particular importancia adquiere la memoria en el paradigma de producción, donde posterior a una situación de ejercicio, la capacidad de retener la información impactará en la reproducción de la actividad futura. A diferencia

Tabla 1. Validez asociada a criterio de las escalas de percepción del esfuerzo en población infantil

\begin{tabular}{|lc|}
\hline $\begin{array}{l}\text { Escalas de percepción } \\
\text { del esfuerzo }\end{array}$ & $\begin{array}{c}\text { Coeficiente de corre- } \\
\text { lación "r" } \mathbf{~ d e ~ P e a r s o n ~}\end{array}$ \\
Escalas sin ilustraciones & 0,69 \\
CR-10 & 0,67 \\
CERT & 0,78 \\
Escalas con ilustraciones & \\
OMNI-Bicicleta & 0,93 \\
OMNI-Marcha-Carrera & 0,81 \\
OMNI-Escalón & 0,89 \\
RPE-C & 0,66 \\
PCERT & 0,81 \\
CALER & 0,90 \\
C-RPES & 0,97 \\
EP-RPE & 0,91 \\
\hline
\end{tabular}

Resultados corresponden al " $r$ " ponderado según tamaño de muestra de acuerdo a los datos publicados por Rodríguez et $\mathrm{al}^{22}$. RPE 6-20: Escala de Borg con categorías de 6 a 20; CR-10: Escala de categoría de Borg de 0 - 10; CERT: Children Effort Rating Table; OMNI: Escalas Omnibus; RPE-C: Escala de Borg 6 - 20 con ilustraciones; PCERT: Pictorial Children Effort Rating Table; CALER: Escala de carro y carga; C-RPES: Escala de esfuerzo percibido para niños; EP-RPE: Escala de esfuerzo percibido de Eston y cols ${ }^{29}$. 
del paradigma de estimación, el cual, se basa en la mera interpretación de un estímulo actual. En virtud de lo anteriormente planteado, es posible establecer que los niños menores a 3 años (etapa sensorio-motriz de Piaget) son incapaces, tanto de interpretar el nivel de PE desde una escala, como producir algún nivel de intensidad de ejercicio a partir de la PE; puesto a que en esta etapa no existe la capacidad para establecer relaciones entre las ilustraciones simbólicas y la intensidad de ejercicio desarrollado ${ }^{1}$.

Entre los 4 y 7 años (período pre-operacional) ha sido observado un incremento en la capacidad para interpretar las escalas de PE (paradigma de estimación $)^{18,30}$. Sin embargo, aun es controversial la validez en la utilización de la PE mediante el paradigma de estimación-producción. Algunas observaciones han concluido que en esta etapa sería eventualmente posible la reproducción de dos a tres niveles de intensidad de fuerza durante una prueba de prensión o de velocidad en cinta rodante $^{31,32}$. Pese a aquello, la evidencia no es lo suficientemente contundente para respaldar la utilización de la PE mediante este paradigma en este grupo etáreo.

Entre los 8 y 12 años (período de operaciones concretas) ha sido establecido que la PE medida a partir de la escala de Borg 6-20 es menor respecto a la de adultos a una misma intensidad relativa de esfuerzo, lo cual, puede ser debido a la escasa validez de esta escala en este grupo de edad $^{9,22}$. Incluso la teoría inicial propuesta por Borg respecto a la estimación de la $\mathrm{FC}$ a partir de la PE (Borg 6-20 multiplicada por 10) carece de validez en niños con este nivel de desarrollo $\operatorname{cognitivo~}^{33,34}$. Por el contrario, las escalas adaptadas para niños han mostrado altos niveles de correlación con $\mathrm{FC} \mathrm{y} \mathrm{VO}_{2}$ en este grupo etario, lo cual, ha sido determinado en diversas modalidades de ejercicios y culturas ${ }^{16,21,35-38}$. Vale la pena destacar que en este grupo etario los niños pueden discriminar niveles de esfuerzo en diferentes partes del cuerpo (piernas, pecho, esfuerzo general $)^{20,39-41}$. Adicionalmente, ha sido observado que en este grupo de niños sería posible la discriminación de más de cuatro niveles de intensidad durante el paradigma de estimaciónproducción ${ }^{16,17,42,43}$.

Entre los 13 y 18 años (período de inteligencia formal) los adolescentes presentan un nivel de abstracción cognitiva que permite una adecuada interpretación de la escala de Borg 6-20 en ambos paradigmas de aplicación ${ }^{6,7}$. No obstante, algunos estudios han mostrado un mejor rendimiento en la medición de la PE a través de escalas adaptadas $^{1,44}$.

\section{Percepción de esfuerzo en niños chilenos: Escala EPinfant}

La medición de la PE requiere de la interpretación de descriptores numéricos, verbales y pictográficos. Por lo tanto, la utilización de una escala adecuadamente construida de acuerdo a las características socio-culturales de la población objetivo es fundamental para un óptimo rendimiento en la medición de esta variable.

En nuestro país fue recientemente desarrollada la escala de medición de esfuerzo percibido infantil (EPInfant) (Figura 2) ${ }^{45}$. En su construcción se empleó una metodología que procuró disminuir los potenciales sesgos que pudiesen afectar sus propiedades psicométricas ${ }^{46}$. Hasta ahora han sido determinadas diversas propiedades de medición. En relación a su validez, presentó un índice de validez de contenido (IVC) superior a $0,90^{46}$. Vale la pena destacar que el IVC es un parámetro determinado en función del porcentaje de acuerdo existente en un panel de expertos respecto a la calidad de la escala ${ }^{47}$. En la validación de la escala EPInfant, el panel de expertos fue constituido por kinesiólogos, psicólogos y médicos; lo cual, permitió establecer su calidad metodológica considerando diversos puntos de vista y campos de aplicación ${ }^{46}$.

En relación a la validez de criterio concurrente, esta escala ha sido evaluada en individuos sanos (eutróficos) de entre 8 y 15 años, durante una prueba de ejercicio incremental. En este contexto, la medición de la PE mediante la escala EPInfant ha sido correlacionada con la FC y carga de trabajo como criterio de referencia, mostrando coeficientes de correlación de Pearson superiores a 0,8 tanto en niños como adolescentes $^{45,46}$. Incluso ha mostrado un rendimiento aceptable en modelos de regresión construidos considerando los datos de todos los individuos de manera simultánea y no solo el promedio de la FC y la PE en cada nivel de intensidad de ejercicio, lo cual, desde el punto de vista estadístico tiende a subestimar la magnitud de la correlación bivariada ${ }^{48}$.

Respecto a la confiabilidad, la escala EPInfant ha sido evaluada empleando tres abordajes estadísticos: cálculo del coeficiente de correlación intraclase ${ }^{49}$, método de Bland-Altman ${ }^{50}$ y determinación de la probabilidad de discordancia, mediante el método de Kaplan Meier aplicado a estudios de confiabilidad (survival agreement $p l o t)^{51}$. Con los tres métodos estadísticos la escala ha mostrado un alto nivel de confiabilidad interobservador en mediciones sucesivas en niños sanos entre 8 y 12 años durante una prueba de 


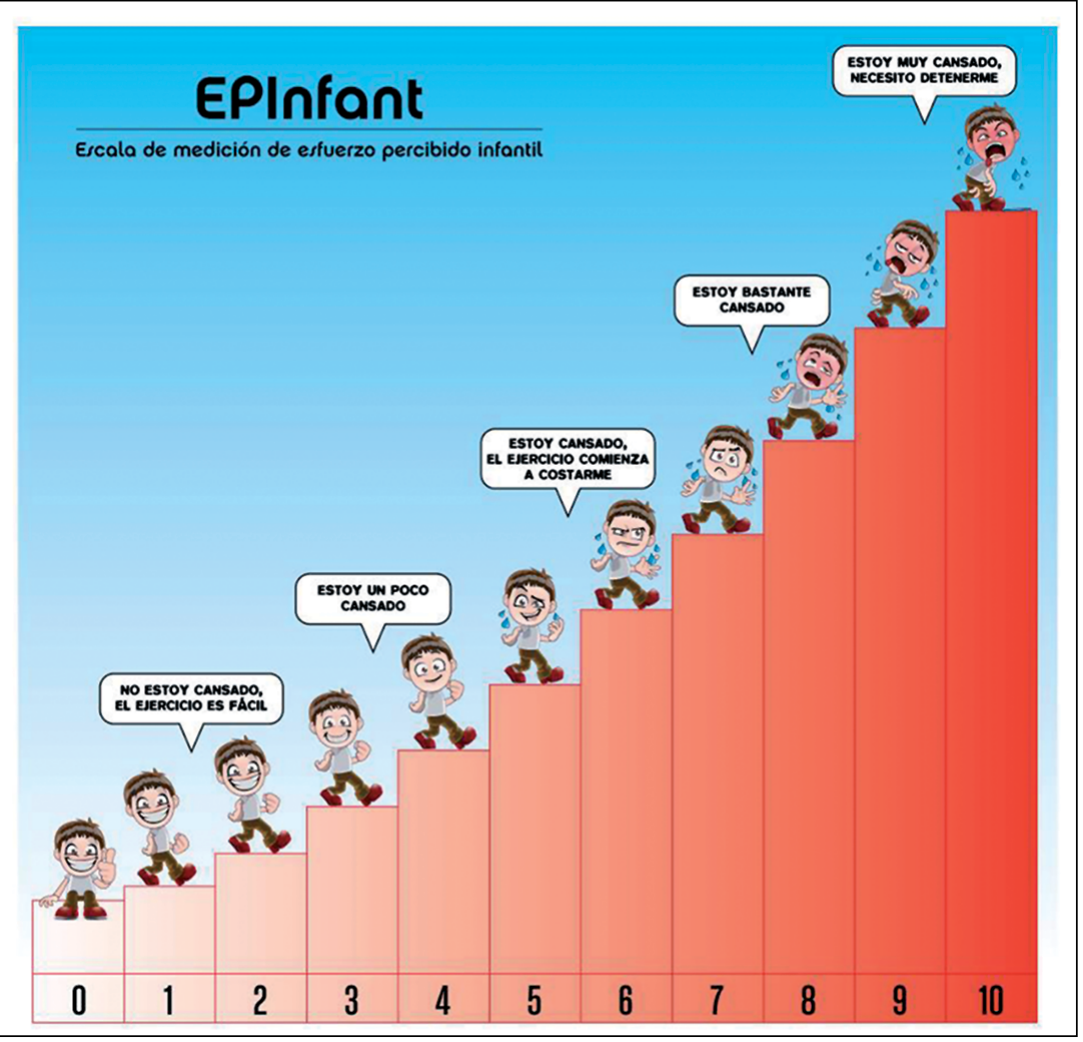

Figura 2. Escala de medición de esfuerzo percibido infantil (EPInfant) para niños chilenos. Imagen modificada de Rodrígue $z^{45}$.

Tabla 2. Índices de validez y confiabilidad de la escala "EPInfant" en niños chilenos

\begin{tabular}{|c|c|c|c|}
\hline & \multicolumn{2}{|c|}{ Validez de criterio } & \multirow{2}{*}{$\begin{array}{c}\text { Confiabilidad } \\
\text { Coeficiente de correlación } \\
\text { intraclase: } \text { CCI }\end{array}$} \\
\hline & $\begin{array}{l}\text { Criterio de referencia } \\
\text { frecuencia cardiaca }\end{array}$ & $\begin{array}{l}\text { Criterio de referencia } \\
\text { carga de trabajo }\end{array}$ & \\
\hline \multicolumn{4}{|c|}{ 13-15 años } \\
\hline Todos & $0,94^{\mathrm{a}} / 0,89^{\mathrm{b}}$ & $0,88^{\mathrm{a}} / 0,86^{\mathrm{b}}$ & ND \\
\hline Niños & $0,93^{\mathrm{a}} / 0,86^{\mathrm{b}}$ & $0,98^{\mathrm{a}} / 0,84^{\mathrm{b}}$ & ND \\
\hline Niñas & $0,95^{\mathrm{a}} / 0,94^{\mathrm{b}}$ & $0,96^{\mathrm{a}} / 0,90^{\mathrm{b}}$ & ND \\
\hline \multicolumn{4}{|c|}{ 8-12 años } \\
\hline Todos & $0,97^{\mathrm{a}}$ & $0,84^{\mathrm{a}}$ & $0,89^{\mathrm{a}}$ \\
\hline Niños & $0,99^{a}$ & $0,98^{\mathrm{a}}$ & $0,88^{\mathrm{a}}$ \\
\hline Niñas & $0,97^{\mathrm{a}}$ & $0,98^{\mathrm{a}}$ & $0,94^{\mathrm{a}}$ \\
\hline
\end{tabular}

ND: No determinado; ${ }^{\text {a }}$ : Modalidad de ejercicio escalón; ${ }^{\text {b}}$ : Modalidad de ejercicio trotadora. Todas las correlaciones son estadísticamente significativas; $\mathrm{p}<0,0001$.

ejercicio incremental en escalón ${ }^{45,48}$. En la Tabla 2 se presentan los coeficientes de validez de criterio y confiabilidad de la escala EPInfant en niños y adolescentes chilenos.

Pese a que la escala EPInfant ha mostrado una validez y confiabilidad aceptable en la población infantil de nuestro país, principalmente mediante el paradigma de estimación, aun se requieren estudios para determinar sus propiedades en el paradigma de estimación-producción, así como también, en niños con enfermedades respiratorias crónicas y en otras modalidades de ejercicio como bicicleta y ejercicios de campo. En este contexto y con el fin de estandarizar su utilización se generaron recomendaciones sobre el uso de esta escala (Tabla 3$)^{46}$. El objetivo final es 
Tabla 3. Recomendaciones para la utilización de la escala "EPInfant" en niños chilenos. Modificado de Rodríguez et al ${ }^{46}$

La escala EPInfant es un instrumento diseñado para cuantificar el nivel de esfuerzo percibido corporal global en niños y adolescentes (menores de 18 años) durante la realización de ejercicio físico

- La escala debe ser inducida antes del inicio del ejercicio físico y debe ser explicada en términos sencillos apropiados a la edad cognitiva del sujeto

- Si el niño/a no sabe leer, se sugiere proporcionar instrucciones dirigidas a la interpretación del esfuerzo percibido, a través de las ilustraciones de niños haciendo ejercicio

- Para una adecuada medición de la percepción de esfuerzo, el sujeto debe contestar la pregunta: ¿Cuán cansado te encuentras durante el ejercicio? La pregunta debe ir dirigida a evaluar la percepción de esfuerzo corporal global incluyendo fatiga de piernas y disnea

A continuación se describe un ejemplo de instrucción apropiada:

- “Antes, durante y después del ejercicio te preguntaré ¿Cuán cansado te encuentras?

- Debes utilizar los números, las palabras o los niños para indicarme tu nivel de cansancio durante la actividad

- Por favor, observa al niño que se encuentra al inicio de la escala, si te sientes como él, significa que no te encuentras cansado

- Por favor, observa a los niños que se encuentra al centro de la escala (niveles 5 y 6), si te sientes como ellos, significa que te encuentras cansado, pero puedes seguir realizando ejercicio

- Por favor, observa al niño que se encuentra al final de la escala, si te sientes como él, significa que te encuentras muy cansado y no puedes seguir realizando ejercicio

- Puedes utilizar cualquiera de los números, frases y/o niños de la escala para decirme cuan cansado te sientes. No existe una respuesta correcta o incorrecta

incentivar el diseño y planificación de estudios ulteriores con metodologías comparables a los estudios ya realizados.

\section{Nuevas hipótesis y perspectivas en el estudio de la percepción de esfuerzo}

Hoy en día existe consenso en que la medición de la PE es una variable que debe ser considerada en la rehabilitación de niños con enfermedades respiratorias y cardiovasculares crónicas, lo cual, ha sido recomendado tanto en Chile como en el extranjero $^{27,52}$. En este sentido, las guías de recomendación vigentes sugieren su medición antes, durante y después de la realización de algún ejercicio físico, ya sea, con fines evaluativos o terapéuticos, considerando el valor absoluto de la $\mathrm{PE}$ un parámetro complementario de estrés cardiorrespiratorio ${ }^{26,27,53}$. Sin embargo, considerando que la PE muestra una fuerte correlación lineal con variables de estrés fisiológico $\left(\mathrm{FC} \mathrm{y} \mathrm{VO}_{2}\right)^{22}$, es probable que la merma en el rendimiento físico debido al sedentarismo, obesidad o existencia de alguna entidad nosológica del sistema cardiorrespiratorio altere la asociación lineal entre estas variables.

Análisis estadísticos secundarios de los datos de estudios ya publicados por nuestro grupo ${ }^{46,48}$, han revelado la existencia de una posible disociación cardio-perceptiva durante el ejercicio, lo cual, mostró asociación con la capacidad física de niños entre 8 y 12 años. En la Figura $3 \mathrm{~A}$ es posible observar la existencia de diferencias significativas en la pendiente de ascenso de la FC en función de la PE al categorizar la muestra de acuerdo a su capacidad física. Estas observaciones motivaron la generación de un índice estadístico de disociación entre la FC y la PE, el cual, equivale a la pendiente (slope) del modelo de regresión entre ambas variables (Figura 3B). Este índice de disociación cardio-perceptiva (IDCP) mostró correlación significativa con el $\mathrm{VO}_{2}$ en la muestra de niños analizada (Figura 3C) 46,48. $^{4}$. Adicionalmente, algunos estudios han sugerido la existencia de cierta disociación entre la respuesta cardiovascular y perceptiva durante la ejecución de pruebas de ejercicio submáximo en niños con bronquiolitis obliterante post-infecciosa ${ }^{54}$; así como también, un reajuste de la PE posterior a programa de rehabilitación pulmonar en niños con enfermedad neuromuscular ${ }^{24}$. No obstante, determinar si el quiebre de la interdependencia entre la PE y variables de estrés fisiológico constituye un marcador predictivo de capacidad física en niños sanos o se asocia con el deterioro 

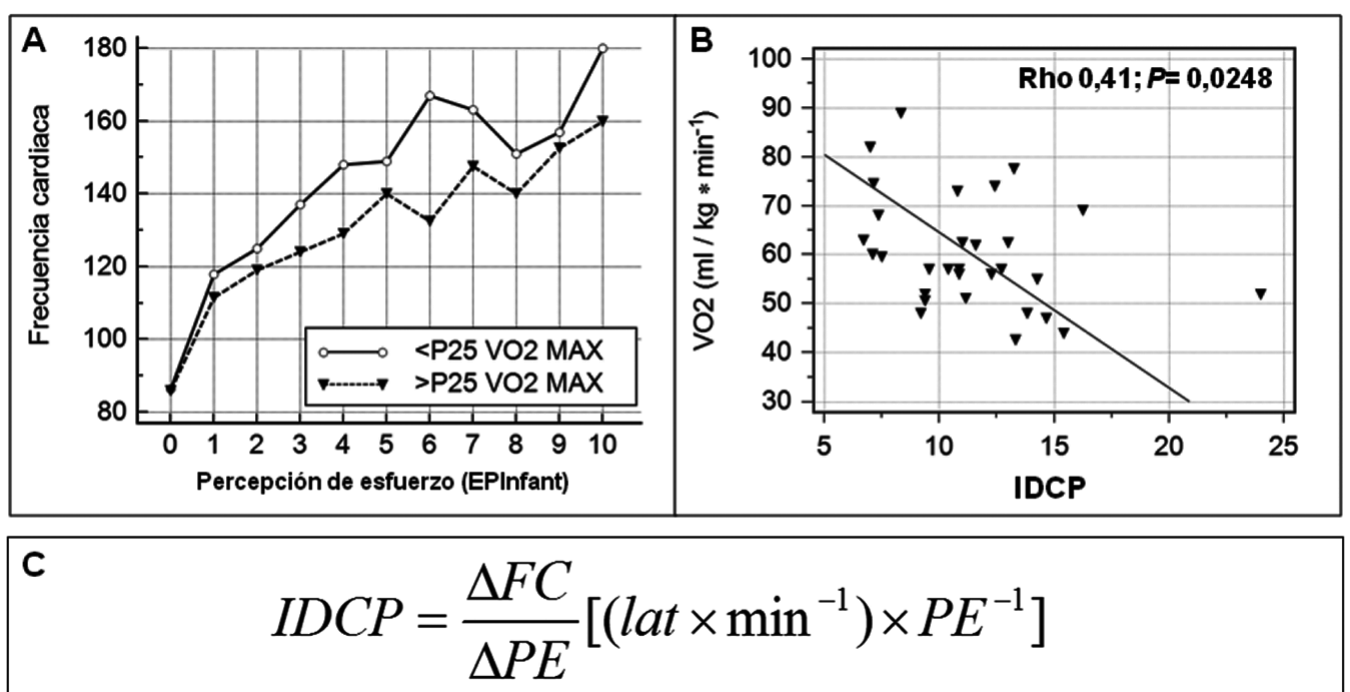

Figura 3. Disociación entre la frecuencia cardiaca y percepción de esfuerzo en niños sanos. En $3 \mathrm{~A}$ se muestra la frecuencia cardíaca en función del esfuerzo percibido en niños categorizados según $\mathrm{VO}_{2}$ máximo considerando al percentil 25 como punto de corte. En 3B se muestra la correlación entre el índice de disociación cardioperceptiva (IDCP) y el $\mathrm{VO}_{2}$ máximo (ml/kg/min) en niños sanos. (Rho; coeficiente de correlación). Los datos fueron obtenidos de 30 niños entre 8 y 12 años durante un test de ejercicio incremental en escalón ${ }^{48}$. En 3C se muestra la ecuación para el cálculo del IDCP, donde $\triangle F C$ corresponde a FC final-FC inicial en latidos cardíacos por min y $\triangle P E$ a la percepción de esfuerzo final-inicial. El IDCP se expresa como (latidos $\left.\bullet \mathrm{min}^{-1}\right) P E^{-1}$.

funcional inducido por el daño cardiopulmonar en niños con enfermedades crónicas, requiere ser verificado en estudios ulteriores.

\section{Conclusiones}

Como ha sido discutido a lo largo del texto la PE durante el ejercicio constituye una variable con propiedades psicométricas apropiadas para ser utilizada en población infantil. La evidencia es contundente en establecer su validez en niños a partir de los 4 años a través de escalas adaptadas y mediante el paradigma de estimación. Sólo a partir de los 8 años es posible sugerir su aplicación mediante el paradigma de estimaciónproducción y desde los 13 a 15 años mediante escalas de adultos como la de Borg 6-20.

Por otra parte, a diferencia de otros instrumentos existentes, la escala EPInfant fue desarrollada mediante un proceso metodológico que consideró aspectos cognitivos y fisiológicos de la respuesta perceptiva durante el ejercicio en niños y adolescentes, lo cual, permite la medición del EP con un alto grado de certeza. Por lo tanto, es posible recomendar su uso en niños entre 8 y 13 años durante el ejercicio dinámico en modalidades de escalón y trotadora. Futuros estudios deben ser realizados para ampliar su utilización en el contexto clínico.

\section{Bibliografía}

1.- GROSLAMBERT A, MAHON A D. Perceived exertion: influence of age and cognitive development. Sports Med. 2006; 36: 911-28.

2.- LAMB K L, ESTON R G. Effort perception in children. Sports Med. 1997; 23: 139-48.

3.- BORG G, DAHLSTRÖM H. Psykofysisk undersökning av arbete pa cykelergometer. Nordisk Medicin 1959; 62 1383-6.

4.- BORG G, DAHLSTRÖM H. The perception of muscular work. Umeä veten. Skapliga skriftserie 1960; 5 1-26.

5.- BORG G. Interindividual scaling and perception of muscular force. Kungl. Fysiol. Sällsk. Förh 1961; 31: $1-26$.

6.- BORG G. Perceived exertion as an indicator of somatic stress. Scand J Rehabil Med 1970; 2: 92-8.

7.- BORG G A. Psychophysical bases of perceived exertion. Med Sci Sports Exerc 1982; 14 : 377-81.

8.- CHEN M J, FAN X, MOE S T. Criterion-related validity of the Borg ratings of perceived exertion scale in healthy individuals: a meta-analysis. J Sports Sci 2002; 20: 873-99.

9.- BAR-OR O. Physical work and effort. En: G. Borg, 
Physical work and effort United Kingdom, Oxford: Pergamon Press 1977; 255-6.

10.- KAHLE C, ULMER H, RUMMEL L. The reproducibility of Borg's RPE scale with female pupils from 7 to II years of age. Eur J Physiol 1977; 368 R26.

11.- ESTON R G, WILLIAMS J G. Exercise intensity and perceived exertion in adolescent boys. Br J Sports Med 1986; 20: 27-30.

12.- WARD O, BLIMKIE C, BAR-OR O. Rating of perceived exertion in obese adolescents. Med Sci Sports Exerc 1986; 18: S72.

13.- GILLACH M C, SALLIS J F, BUONO M J, PATTERSON P, NADER P R. The relationship between perceived exertion and heart rate in children and adults. Pediatr Exerc Sci 1989; 1: 360-8.

14.- WARD D S, BAR-OR O. Use of the Borg scale in exercise prescription for overweight youth. Can J Sport Sci 1990; 15: 120-5.

15.- LAMB K L. Children's ratings of effort during cycle ergometry: an examination of the validity of two effort rating scales. Pediatr Exerc Sci 1995; 7: 407-21.

16.- LAMB K L. Exercise regulation during cycle ergometry using the children's effort rating table (CERT) and rating of perceived exertion (RPE) scales. Pediatr Exerc Sci 1996; 8 337-50.

17.- ESTON R G, LAMB K L, BAIN A, WILLIAMS A M, WILLIAMS J G. Validity of a perceived exertion scale for children: a pilot study. Percept Mot Skills 1994; 78 : 691-7.

18.- WILLIAMS J G, ESTON R, FURLONG B. CERT: a perceived exertion scale for young children. Percept Mot Skills 1994; 79 (3 Pt 2): 1451-8.

19.- YELLING M, LAMB K L, SWAINE I. Validity of a pictorial perceived exertion scale for effort estimation and effort production during stepping exercise in adolescent children. Eur Phy Educ Rev 2002; 8: 157-75.

20.- ROEMMICH J N, BARKLEY J E, EPSTEIN L H, LOBARINAS C L, WHITE T M, FOSTER J H. Validity of PCERT and OMNI walk/run ratings of perceived exertion. Med Sci Sports Exerc 2006; 38: 1014-9.

21.- MARINOV B, MANDADJIEVA S, KOSTIANEV S. Pictorial and verbal category-ratio scales for effort estimation in children. Child Care Health Dev 2008; 34 : $35-43$.

22.- RODRÍGUEZ I, ZAMBRANO L, MANTEROLA C. Criterion-related validity of perceived exertion scales in healty children: a systematic review and meta-analysis. Arch Argent Pediatr 2016; 114: 120-8.

23.- FAULKNER J, ESTON R. Perceived exertion research in the 21 st century: Developments, reflections and questions for the future. J Exerc Sci Fit 2008; 6: 1-14.

24.- RODRÍGUEZ I, FUENTES C, RIVAS C, MOLINA F, SEPÚLVEDA C, ZENTENO D. Rehabilitación respiratoria en el paciente neuromuscular: efectos sobre la tolerancia al ejercicio y musculatura respiratoria. Resultado de una serie de casos. Rev Chil Enferm Respir
2013; 29: 196-203.

25.- RODRÍGUEZ I, ZENTENO D. Ventilación no invasiva durante el ejercicio en niños con bronquiolitis obliterante post infecciosa. A propósito de un caso. Neumol Pediatr 2014; 9: 27-30.

26.- ZENTENO D, PUPPO H, GONZÁLEZ R, KOGAN R. Test de marcha de 6 minutos en pediatría. Neumol Pediatr 2007; 2: 109-14.

27.- ZENTENO D, PUPPO H, VERA R, TORRES R, KUO C-Y, SALINAS P, et al. Guías de rehabilitación para niños con enfermedades respiratorias crónicas. Neumol Pediatr 2007; 3 (Supl 1): 25-33.

28.- PARFITT G, SHEPHERD P, ESTON R G. Reliability of effort production using the children's CALER and BABE perceived exertion scales. J Exerc Sci Fit 2007; 5: 49-55.

29.- ESTON R G, PARFITT G, CAMPBELL L, LAMB K L. Reliability of effort perception for regulating exercise intensity in children using the Cart and Load Effort Rating (CALER) Scale. Pediatr Exerc Sci 2000; 12: 388-97.

30.- GROSLAMBERT A, HINTZY F, HOFFMAN M D, DUGUE B, ROUILLON J D. Validation of a rating scale of perceived exertion in young children. Int J Sports Med 2001; 22: 116-9.

31.- GROSLAMBERT A, NACHON M, ROUILLON J $D$. Influence of the age on self regulation of static grip forces from perceived exertion values. Neurosci Lett 2002; 325: 52-6.

32.- GROSLAMBERT A, MONNIER BENOIT P, GRANGE C, ROUILLON J D. Self-regulated running using perceived exertion in children. J Sports Med Phys Fitness 2005; 45: 20-5.

33.- DUNCAN G, MAHON A, GAY J, SHERWOOD J. Physiological and perceptual responses to graded treadmill and cycle exercise in male-children. Pediatr Exerc Sci 1996; 8: 251-8.

34.- Mahon A D, Marsh M L. Reliability of the rating of perceived exertion at ventilatory threshold in children. Int J Sports Med 1992; 13: 567-71.

35.- LEUNG M L, CHUNG P K, LEUNG R W. An assessment of the validity and reliability of two perceived exertion rating scales among Hong Kong children. Percept Mot Skills 2002; 95 (3 Pt 2): 1047-62.

36.- CASSADY S L, KAUFMAN B A, KELLY C E, EISENMANN S C, WENTZIEN J M. Validity of a New Perceived Exertion Scale for Children. Cardiopulmonary Phys Ther J 1998; 9: 3-8.

37.- SUMINSKI R R, ROBERTSON R J, GOSS F L, OLVERA N. Validation of the Omni Scale of Perceived Exertion in a sample of Spanish-speaking youth from the USA. Percept Mot Skills 2008; 107: 181-8.

38.- LEUNG F P, YUNG L M, LAHER I, YAO X, CHEN Z Y, HUANG Y. Exercise, vascular wall and cardiovascular diseases: an update (Part 1). Sports Med 2008; 38 : 1009-24. 
39.- BARKLEY J E, ROEMMICH J N. Validity of the CALER and OMNI-bike ratings of perceived exertion. Med Sci Sports Exerc 2008; 40: 760-6.

40.- ROBERTSON R J, GOSS F L, ANDREACCI J L, DUBE J J, RUTKOWSKI J J, SNEE B M, et al. Validation of the children's OMNI RPE scale for stepping exercise. Med Sci Sports Exerc 2005; 37 (2): 290-8.

41.- ROBERTSON R J, GOSS F L, BOER N F, PEOPLES J A, FOREMAN A J, DABAYEBEH I M, et al. Children's OMNI scale of perceived exertion: mixed gender and race validation. Med Sci Sports Exerc 2000; 32: 452-8.

42.- WILLIAMS J G, ESTON R G, STRETCH C. Use of the rating of perceived exertion to control exercise intensity in children. Pediatr Exerc Sci 1991; 3: 21-7.

43.- WARD D, JACKMAN J, GALIANO F. Exercise intensity reproduction: Children versus adults. Pediatr Exerc Sci 1991; 3: 209-18.

44.- PFEIFFER K A, PIVARNIK J M, WOMACK C J, REEVES M J, MALINA R M. Reliability and validity of the Borg and OMNI rating of perceived exertion scales in adolescent girls. Med Sci Sports Exerc 2002; 34: 2057-61.

45.- RODRÍGUEZ I. Escala de medición de esfuerzo percibido infantil (EPInfant): validación en niños y adolescentes chilenos. Rev Chil Pediatr 2015; en prensa. DOI 10.1016/j.rchipe.2015.09.001.

46.- RODRÍGUEZ I, ZENTENO D, CISTERNAS L, RODRÍGUEZ P, REYES G, TRONCOSO K. Construcción y evaluación de EPInfant: una escala para la medición del esfuerzo percibido en población pediátrica. Arch Argent Pediatr 2015; 113: 550-7.

47.- POLIT D F, BECK C T, OWEN S V. Is the CVI an acceptable indicator of content validity? Appraisal and recommendations. Res Nurs Health 2007; 30: 459-67.

48.- RODRÍGUEZ I, MANTEROLA C. Validación inicial de la escala de medición de esfuerzo percibido infantil (EPInfant) en niños chilenos. Biomédica 2016; 36: 29 38.

49.- WEIR J P. Quantifying test-retest reliability using the intraclass correlation coefficient and the SEM. J Strength Cond Res 2005; 19: 231-40.

50.- GIAVARINA D. Understanding Bland Altman analysis. Biochem Med (Zagreb) 2015; 25: 141-51.

51.- LUIZ R R, COSTA A J, KALE P L, WERNECK G L. Assessment of agreement of a quantitative variable: a new graphical approach. J Clin Epidemiol 2003; 56: 963-7.

52.- TAKKEN T, GIARDINI A, REYBROUCK T, GEWILLIG M, HOVELS-GURICH H H, LONGMUIR P E, et al. Recommendations for physical activity, recreation sport, and exercise training in paediatric patients with congenital heart disease: a report from the Exercise, Basic \& Translational Research Section of the European Association of Cardiovascular Prevention and Rehabilitation, the European Congenital Heart and Lung Exercise Group, and the Association for European Paediatric Cardiology. Eur J Prev Cardiol 2012; 19: 1034-65.

53.- VEGA-BRICEÑO L, ZENTENO D. Guía clínica para el diagnóstico y cuidado de niños/adolescentes con bronquiolitis obliterante post-infecciosa, 2009. Rev Chil Enf Respir 2009; 25: 141-63.

54.- RODRÍGUEZ I, HENRÍQUEZ S, VÁSQUEZ P, ZENTENO D. Test de caminata de seis minutos y función pulmonar en pacientes con bronquiolitis obliterante post infecciosa. Rev Chil Enferm Respir 2014; 30: 68-74.

Correspondencia a:

Klgo. Iván Rodríguez Núñez

Facultad de Ciencias de la Salud,

Universidad San Sebastián.

Lientur $n^{\circ} 1457$ Concepción, Chile.

Email: ivan.rodriguez@uss.cl 